\title{
Direction and time dependent fluxes with AMS-02
}

\author{
Iris Gebauer* \\ Karlsruhe Institute of Technology, Institut für Experimentelle Kernphysik, D-76131 Karlsruhe, \\ Germany
}

\author{
Stefan Zeissler, Karen Andeen; Nikolay Nikonov, Valerio Vagelli; Carmen Merx \\ Karlsruhe Institute of Technology, Institut für Experimentelle Kernphysik, D-76131 Karlsruhe, \\ Germany \\ E-mail: stefan.zeissler@kit.edu
}

The Alpha Magnetic Spectrometer (AMS-02) is a state-of-the-art particle detector designed to operate as an external module on the International Space Station (ISS). In this unique space environment cosmic particles can be measured with high precision over an energy range from $\mathrm{GeV}$ up to a few TeV. In 2014, the AMS collaboration provided precise measurements of the electron and positron fluxes, which indicate an additional source of positrons among the various cosmic particles. The arrival directions of energetic electrons and positrons in the range of hundreds of GeVs convey fundamental information on their origin, whereas low energy electrons and positrons in the $\mathrm{MeV}$ to tens of $\mathrm{GeV}$ range are subject to time dependent solar modulation.

We evaluate the AMS-02 detector acceptance as a function of incoming angle in the detector frame. Using the detector pointing information over the entire AMS-02 data taking period, we build time-dependent sky maps in galactic coordinates of the incoming directions of the electrons and positrons, as well as time-dependent reference maps. The latter simulate the expected AMS-02 measurement of isotropic fluxes. Our maps allow us to study possible anisotropies in the arrival directions of energetic electrons and positrons, as well as time and direction dependent effects at low energies due to the influence of the solar wind. This technique can be applied to all cosmic ray species. We demonstrate the performance of the method using AMS-02 data.

The 34th International Cosmic Ray Conference,

30 July- 6 August, 2015

The Hague, The Netherlands

\footnotetext{
*Speaker.

${ }^{\dagger}$ Now at Marquette University, 1250 W. Wisconsin Avenue, Milwaukee, WI 53233, USA.

${ }^{\ddagger}$ Now at INFN - Sezione di Perugia,06123 Perugia, Italy.
} 


\section{Introduction}

Cosmic ray (CR) electrons and positrons have been the object of intensive studies by the AMS collaboration [1][2][3][4] and other experiments [5][6]. Complementary to protons, their fluxes provide fundamental information about CR acceleration processes and CR sources as well as their propagation mechanisms. The typical propagation length of electrons and positron is of the order of kpc at energies of $100 \mathrm{GeV}$ due to fast energy losses via synchrotron radiation and inverse Compton scattering. This means the sources of energetic electrons and positrons have to be comparably close to the Sun. Variations in the arrival directions of energetic electrons and positrons therefore may provide valuable information about their origin and the properties of the local interstellar environment [7]. For energies up to about $30 \mathrm{GeV}$ CR fluxes are modulated by the time dependent solar wind. Solar particles can be observed directly with neutron monitors, which provide data up to a few hundreds of $\mathrm{MeV}$. With its large acceptance up to $\mathrm{TeV}$ energies the Alpha Magnetic Spectrometer (AMS-02) can be used to monitor the impact of solar activity on CR fluxes up to energies of hundreds of $\mathrm{GeV}$.

Here we present a method to measure CR fluxes as a function of time and incoming direction. The method can be applied to all CR species, thus enabling us to study the time and directional dependence of solar events in the $\mathrm{GeV}$ range, as well as posssible anisotropies in CR incoming directions at high energies. This allows searches for possible point sources of CRs, which might contribute to the observed rise in the fluxes of positrons [3] and protons [8][9].

\section{The AMS-02 Detector}

Figure 1 shows the layout of the AMS02 detector. It consists of 7 precision silicon tracker planes inside the magnetic field of a permanent magnet (inner tracker), plus two additional planes, one on top of a transition radiation detector (TRD) and one on top of an electromagnetic calorimeter (ECAL). A time-offlight detector (TOF) consisting of 4 scintillation counter planes, 2 above the inner tracker and 2 below, is used as the primary trigger. The inner tracker is surrounded by an anti-coincidence counter (ACC). The detector is completed by a ring imaging cherenkov detector ( $\mathrm{RICH})$ for the measurement of heavy nuclei. A detailed detector description can be found in [1].

The TRD is designed to identify electrons and positrons among the numerous protons by virtue of transition radiation emitted in a fiber fleece radiator and collected in 20 layers of proportional tubes filled with a 90:10 Xe:CO2 mixture. Tran-

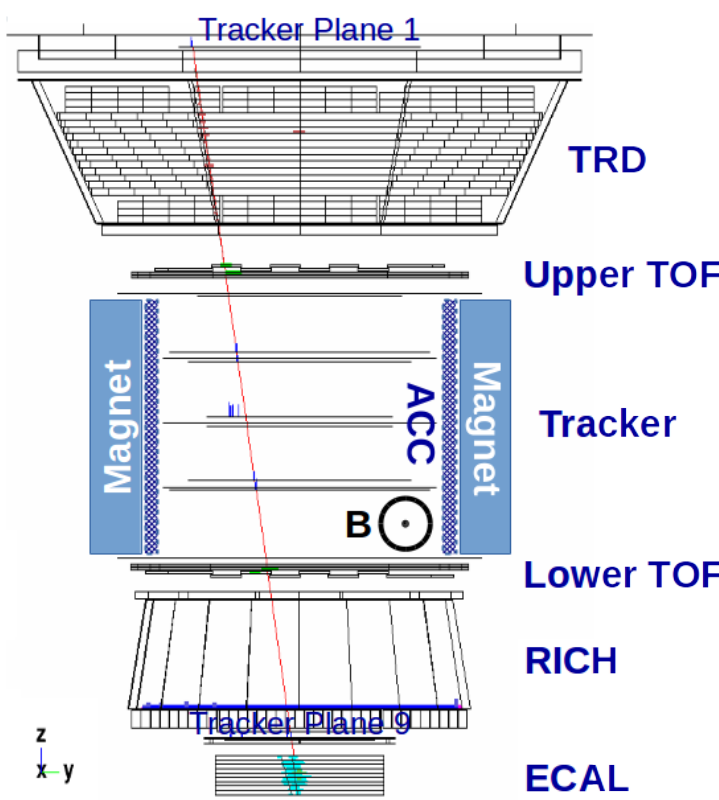

Figure 1: AMS-02 Event display of a 93 $\mathrm{GeV}$ electron. 
sition radiation and ionization signals are combined in a log-likelihood estimator. The ECAL, which has a thickness corresponding to 17 radiation lengths, discriminates between electrons and protons by virtue of the three-dimensional shower shape. Variables describing the shower shape are combined into a boosted decision tree (BDT). Together, the TRD and ECAL can select electrons and positrons and efficiently reject protons to a power of 3 from a charge $Z=1$ particle sample defined by tracker and TOF defined as described in [4].

\section{The Flux Measurement}

The flux of CR particles in units of $\mathrm{GeVm}^{-2} \mathrm{sr}^{-1} \mathrm{~s}^{-1}$ as function of energy and the incoming direction in galactic longitude $\varphi$ and latitude $\theta$ is given by

$$
\Phi(\theta, \varphi, E+\Delta E)=\frac{N(\theta, \varphi, E+\Delta E)}{A(E) \varepsilon_{S e l} T_{E x p}(\theta, \varphi, E+\Delta E) \Delta E},
$$

where $N$ is the number of particles within the energy interval $E+\Delta E$ in $\mathrm{GeV}$ coming from said direction in galactic sky, $A$ is the effective detector acceptance with trigger efficiency after the charge $Z=1$ selection in units of $\mathrm{m}^{-2} \mathrm{sr}^{-1}, \varepsilon_{S e l}$ is the particles selection efficiency by ECAL and TRD and $T_{E x p}(\theta, \varphi, E)$ is the exposure time in the galactic sky in seconds. The acceptance $A$ depends on the incoming direction in the detector frame, while the exposure time is a function of the galactic pointing direction.

\subsection{Event Selection}

The goal of this study is a time and direction dependent flux measurement. To accomplish this, the single particle information needs to be preserved for each event at each stage of the selection. We use a cut-based selection and use template fits to evaluate the level of remaining background contamination. Electrons and positrons are selected with high purity using ECAL and TRD. To control the proton dominated background template fits in the TRD estimator variable are performed. This is done in two steps:

(i) An efficient cut on the ECAL BDT is applied and the signal over background ratio is evaluated through template fits in the TRD estimator variable.

(ii) The TRD estimator distribution is scanned for all selection cuts and a cut is fixed in a way that the background is $\ll 1 \%$.

In Figure 2 the procedure is illustrated. With a targeted signal to background ratio of $10^{3}$ a selection efficiency $\varepsilon_{S e l}$ of about $75 \%$ up to $100 \mathrm{GeV}$ can be achieved.

\subsection{Selection Efficiency}

The selection efficiencies are evaluated as a function of the geomagnetic cutoff defined within the International Geomagnetic Reference Field (IGRF) model for every selection cut. The geomagnetic cutoff determines the rate of low rigidity particles. These particles are deflected by the Earth's magnetic field at the equator and reach the detector at the poles only. We verify that this difference in particle rates does not have an impact on the selection efficiency. Figure 3 shows the 

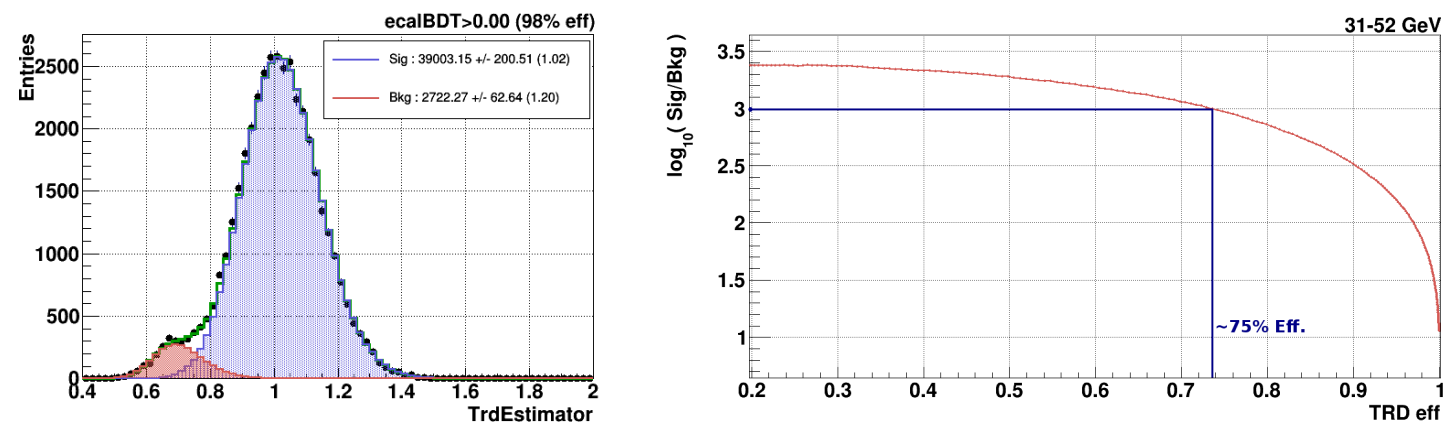

Figure 2: Left: Template fit in TRD estimator variable after a cut on ECAL BDT has been applied. Right: Evaluated signal over background ratio from template fit.
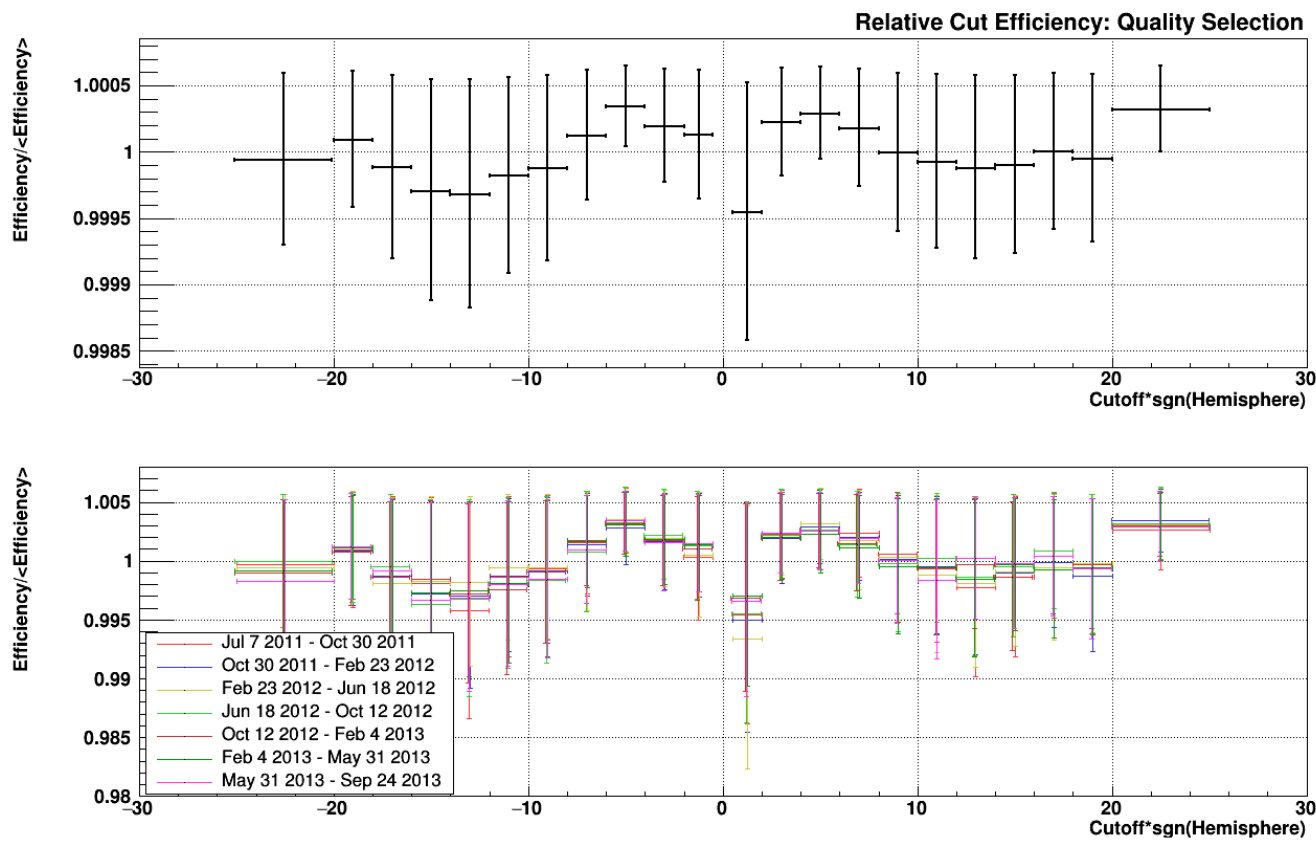

Figure 3: Relative efficiency with respect to mean efficiency of event quality cuts in the energy range of $20-30 \mathrm{GeV}$ as a function of IGRF cutoff multiplied by the sign of the Earth's hemisphere (top), and separated into time intervals (bottom).

relative efficiency of the quality cuts as a function of geomagnetic cutoff multiplied by the sign of the Earth's hemisphere, where the southern hemisphere has negative sign. Low absolute values correspond to the polar region with high particle rate while higher absolute values correspond to a position close to equator. No dependence of the selection efficiency on the geomagnetic cutoff could be found. It is therefore considered to be constant for every detector position and pointing direction in the galactic sky. Also the selection efficiency is shown in different time periods to verify that we don't have to consider time dependent variations. However this does not hold for the trigger efficiency which has to be studied separately. 


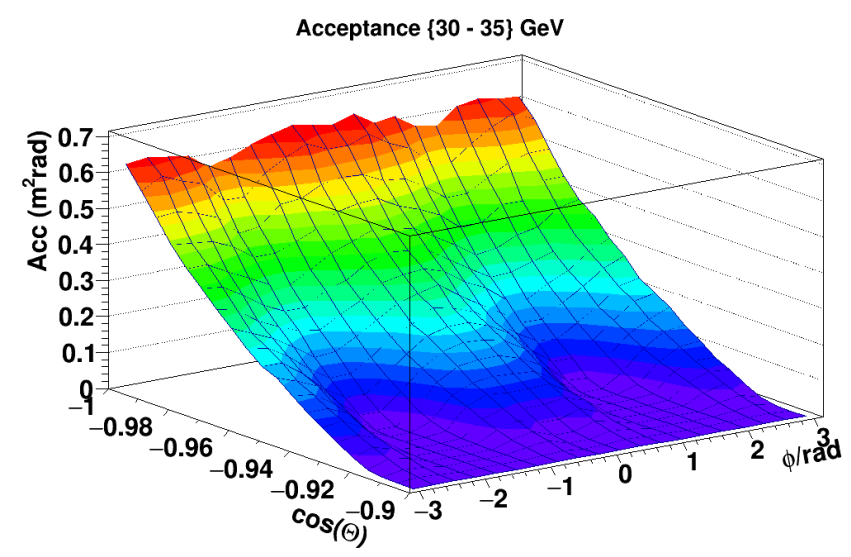

Figure 4: Acceptance for electrons as function of the incoming direction in the detector frame for the energy range $30-35 \mathrm{GeV}$.

\subsection{Acceptance}

The acceptance is evaluated as a function of the electron energy as well as incoming direction in detector coordinates from full AMS-02 Monte Carlo simulations according to

$$
\operatorname{Acc}\left(E+\Delta E, \theta_{D}, \phi_{D}\right)=A_{e f f} \times \frac{N_{S e l}\left(E+\Delta E, \theta_{D}, \phi_{D}\right)}{N_{G e n}\left(E+\Delta E, \theta_{D}, \phi_{D}\right)},
$$

where $A_{\text {eff }}$ is the effective area of the plane above the detector from where electrons are generated uniformly into a sector with solid angle $\Omega=2 \pi . N_{S e l}$ and $N_{G e n}$ are the number of selected and generated events in bins of the incoming direction. The acceptance template as a function of incoming direction in detector coordinates $\left(\theta_{D}, \phi_{D}\right)$ is shown in Figure 4 . This acceptance template is used for the creation of the time dependent reference map.

\subsection{Time Dependent Reference Map and Exposure Time}

In order to get the measurement time in a specific region in the galactic sky at a given time the detector acceptance as function of the incoming direction in the detector frame (as described in 3.3) is projected to the galactic sky and weighted with the detector livetime for each second of data taking. In this way, a map of the expected particle rate for perfectly isotropic particle arrival directions is obtained. The map can be renormalized to calculate the measurement time in galactic sky coordinates. From this map the measurement time, exposure, and acceptance in specific regions of the sky and in specific measurement periods can be retrieved. Due to the Earth's magnetic field this map is energy dependant as already described in 3.2. The resulting map at ISS altitude for electrons with an energy above $40 \mathrm{GeV}$ for the first 2.5 years of data taking can be seen on the left hand side in Figure 5. The integration over the the entire data taking time yields a map of a perfectly isotropic sky as it would be seen by AMS-02. Such maps can be used as a reference map for anisotropy searches as discussed in [11]. On the right hand side a comparison by significance as defined in [10] of the deviation of the reference map from measured electrons in the same time period is shown. These comparisons of the reference map to data can also provide an estimation of systematic uncertainties of the method. 


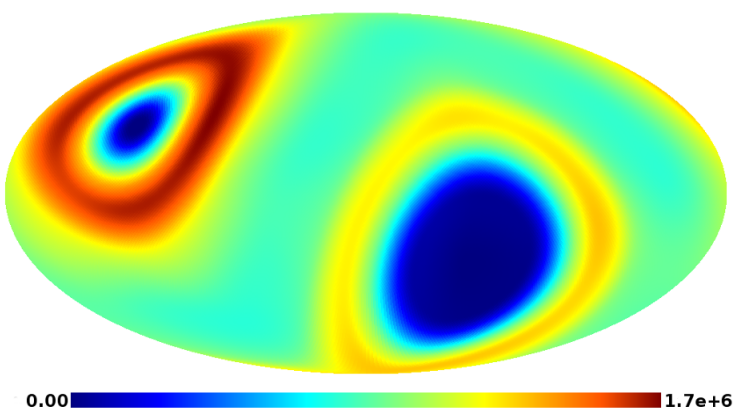

Time in seconds inside FOV

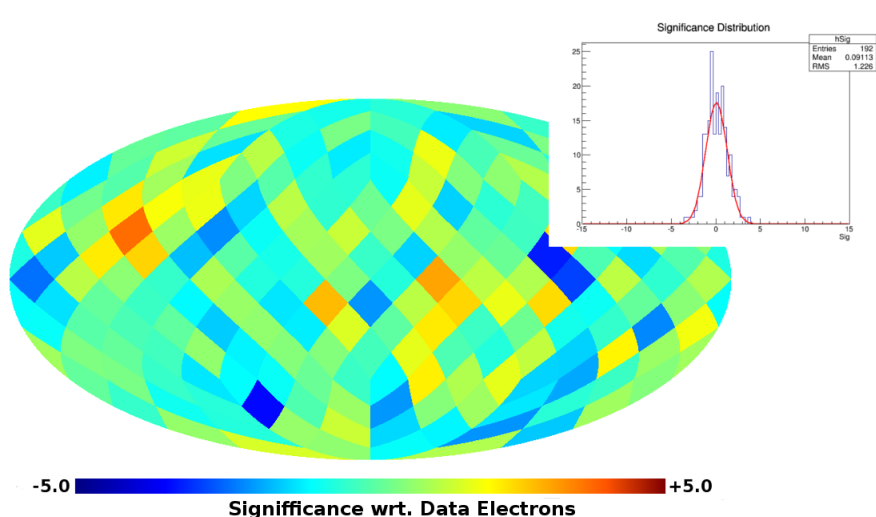

Signifficance wrt. Data Electrons

Figure 5: Left: Integrated reference map in galactic sky weighted by measuring time at ISS altitude in the period of May 2011 to November 2014 for electrons with energy greater than $40 \mathrm{GeV}$. Right: Significance of the deviation of the data electrons from the reference map, with energy greater than $40 \mathrm{GeV}$ and histogram of the significance distribution with fitted Gaussian.

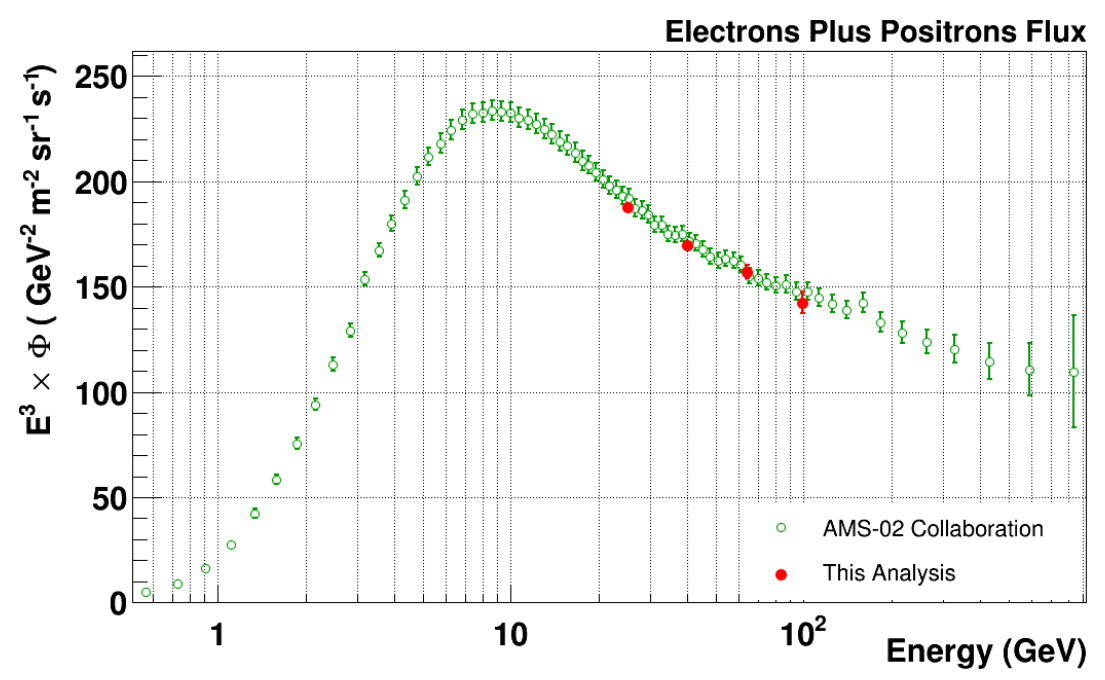

Figure 6: Comparison of the published AMS-02 combined electron and positron flux [4] to the flux from the cut based strategy presented in this analysis (statistical errors only).

\section{Results}

In Figure 6 the flux of electrons plus positrons calculated with the analysis strategy presented here is shown in comparison to the published AMS-02 result [4]. With the cut based analysis electrons and positrons are selected with an efficiency of about $75 \%$ with respect to a pure template fit method as used in the official result. As discussed in Section 3.1, the cut-based approach is necessary to conserve single particle information. A deeper understanding of systematic uncertainties of the method such as the stability of the number of particles over the calculated selection efficiencies using different sets of selection cuts has yet to be done. The width of the binning in energy measured by ECAL is chosen by the statistics available in a certain incoming direction and time and is well above the ECAL energy resolution. Therefore migration of signal events in neighbouring 
energy bins can be neglected. Also the sensitivity of the method by testing artificial signals in different sky directions has to be stated.

\section{Conclusion}

A method to obtain the electron plus positron flux in time as well as incoming direction in galactic coordinates up to an energy of $350 \mathrm{GeV}$ has been presented. Particles are identified with the AMS-02 ECAL and TRD in a background free approach keeping single particle information such as the incoming coordinates and time. The selection can be controlled with a TRD template fit method similar to the one used in several AMS-02 analysis publications such as [4]. The measuring time in specific sky directions is evaluated through a time dependent reference map using a acceptance estimate calculated from Monte Carlo data. The method can easily be adapted to other particle species such as protons.

The method presented here may be used to study the sources of cosmic particles as well as the time dependent impact of low energy solar particles on CRs in the Earth's magnetic field. An in-depth study of systematics originating from the acceptance template as well as the time and direction resolution of the method is ongoing.

Our reference map resembles a measurement of an isotropic sky at ISS position. If CR arrival directions are isotropic in the interstellar medium, a possible distortion of CR arrival directions at ISS orbit is expected due to the Earth's magnetic field. For example, for electrons with an energy of $100 \mathrm{GeV}$ the incoming direction can change by up to 40 deg due to deflections in the magnetosphere over a distance of 10 Earth radii. This distortion needs to be taken into account in physics analyses of particle arrival directions: Particle trajectories can be backtraced in the Earth's magnetic field up to the magnetospheric border were the arrival directions of CRs may be assumed to be isotropic. Consequently, the method presented here needs to be applied to a sample of "forward traced" particles (back traced particles of opposite charge), which is assumed to be isotropic outside the influence of the magnetophere.

\section{References}

[1] M. Aguilar et al (AMS Collaboration), First Result from the Alpha Magnetic Spectrometer on the International Space Station: Precision Measurement of the Positron Fraction in Primary Cosmic Rays of 0.5-350 GeV, Phys. Rev. Lett. 110, 141102 - Published 3 April 2013

[2] L. Accardo et al (AMS Collaboration), High Statistics Measurement of the Positron Fraction in Primary Cosmic Rays of 0.5-500 GeV with the Alpha Magnetic Spectrometer on the International Space Station, Phys. Rev. Lett. 113, 121101 - Published 18 September 2014

[3] M. Aguilar et al (AMS Collaboration), Electron and Positron Fluxes in Primary Cosmic Rays Measured with the Alpha Magnetic Spectrometer on the International Space Station, Phys. Rev. Lett. 113, 121102 - Published 18 September 2014

[4] M. Aguilar et al (AMS Collaboration), Precision Measurement of the $\left(e^{+}+e^{-}\right)$Flux in Primary Cosmic Rays from $0.5 \mathrm{GeV}$ to $1 \mathrm{TeV}$ with the Alpha Magnetic Spectrometer on the International Space Station, Phys. Rev. Lett. 113, 121102 - Published 26 November 2014 
[5] M.Ackerman et al, Measurement of Separate Cosmic-Ray Electron and Positron Spectra with the Fermi Large Area Telescope, Phys. Rev. Lett. 108, 011103 - Published 5 January 2012

[6] S.Torii et al, High-energy electron observations by PPB-BETS flight in Antarctica, arXiv:0809.0760 [astro-ph]

[7] T. Delahaye et al, Galactic electrons and positrons at the Earth:new estimate of the primary and secondary fluxes, Astron. Astrophys. 524 (2010) A51

[8] M. Aguilar et al. (AMS Collaboration), Precision Measurement of the Proton Flux in Primary Cosmic Rays from Rigidity 1 GV to 1.8 TV with the Alpha Magnetic Spectrometer on the International Space Station, Phys. Rev. Lett. 114, 171103 - Published 30 April 2015

[9] Adriani et al., PAMELA Measurements of Cosmic-Ray Proton and Helium Spectra, Science, 332 (6025), 69-72 - Published online 3 March 2011

[10] T. Li and Y. Ma, Analysis Methods for Results in Gamma-Ray Astronomy, AJ, 272:317-324 Published 1 September 1983

[11] I. Gebauer, K. Andeen, N. Nikonov, V. Vagelli, and S. Zeissler, Methods for cosmic ray anisotropy searches with $A M S-02$, this volume 